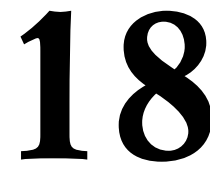

\title{
EVALUACIÓN DE ENTORNOS INMERSIVOS 3D COMO HERRAMIENTA DE APRENDIZAJE B-LEARNING*
}

\section{(EVALUATION OF 3D IMMERSIVE ENVIRONMENTS IN B-LEARNING IMPLEMENTATIONS)}

Óscar González-Yebra

Manuel A. Aguilar

Fernando J. Aguilar

Manuel Lucas

Universidad de Almería

DOI: $10.5944 / e d u c X X 1.16204$

\section{Cómo referenciar este artículo/How to reference this article:}

González-Yebra, Ó., Aguilar, M.A., Aguilar, F.J. y Lucas, M. (2018). Evaluación de entornos inmersivos 3D como herramienta de aprendizaje B-Learning. Educación XX1, 21(2), 417-440, doi: $10.5944 /$ educXX1.16204

González-Yebra, Ó., Aguilar, M.A., Aguilar, F.J. \& Lucas, M. (2018). Evaluación de entornos inmersivos 3D como herramienta de aprendizaje B-Learning. [Evaluation of 3D immersive environments in B-Learning implementations]. Educación XX1, 21(2), 417-440, doi: 10.5944/educXX1.16204

\section{RESUMEN}

El presente trabajo se ha desarrollado en el marco de un proyecto de innovación educativa para la adaptación de la Universidad de Almería al EEES. Tiene como objetivo general el estudio del potencial de los entornos inmersivos 3D para la enseñanza-aprendizaje a distancia. En este sentido se ha evaluado el uso de la plataforma Second Life para complementar y reforzar las clases presenciales de la asignatura Diseño Asistido por Ordenador mediante una alternativa eficaz y ágil para la comunicación a distancia a través de la WWW, manteniendo, en la medida de lo posible, el sentimiento de pertenencia a una clase de todas las personas participantes. 
El estudio se llevó a cabo a lo largo de tres fases diferenciadas. En la primera, se diseñó y planificó el procedimiento para la investigación experimental. En la segunda, se llevaron a cabo las diferentes sesiones programadas en el marco del proyecto y se procedió a la obtención de las valoraciones del alumnado. En la tercera fase se adaptó y aplicó el conocido modelo de Kano para obtener una evaluación cuantitativa de la satisfacción del grupo de estudiantes tanto con la interfaz del entorno inmersivo 3D empleado como con el propio método de enseñanza síncrona a distancia.

En líneas generales la respuesta del alumnado participante en relación a la componente teórica de la asignatura ha sido ampliamente positiva, inclinándose por una docencia presencial en el caso de la componente puramente práctica o aplicada. Los hallazgos obtenidos en este estudio pueden ser de utilidad para el profesorado/personal investigador interesado en el diseño y recreación de aulas virtuales análogas, así como para avanzar en estrategias que promuevan de una forma efectiva la enseñanza B-Learning apoyada en entornos inmersivos 3D.

\section{PALABRAS CLAVE}

Enseñanza Semipresencial; Innovación Docente; Educación Superior; Second Life; Satisfacción Estudiantes; Modelo de Kano.

\section{ABSTRACT}

This work has been developed within the framework of a project of educational innovation aimed at adapting the University of Almeria to the European Higher Education Area. Its general objective was to investigate the feasibility of applying 3D immersive environments for distance teaching and learning. In this sense, the use of the widely known platform Second Life has been evaluated with regards to efficiently complementing the traditional faceto-face learning method applied in the subject of Computer Aided Design, maintaining insofar as possible the feeling of belonging to a classroom for all the classmates involved.

The study was conducted throughout three different phases. In the first one, the procedure for experimental research was designed and planned. In the second stage, several working sessions focused on putting the proposed method into practice were carried out, later obtaining the opinion of the students through a specific questionnaire. Within the third phase, an adapted version of the well-known Kano's model was applied to quantitatively assess student satisfaction with both the 3D immersive environment interface and the synchronous and distance learning method tested.

Overall, the student response in relation to the theoretical component of the subject has been broadly positive, although they clearly preferred face-to-face 
teaching in the case of the purely practical component (CAD laboratory). The findings obtained in this study may be useful for teachers/researchers who are interested in the design of similar virtual classrooms. It can also help progress towards effective strategies for promoting the use of B-Learning experiences supported by 3D immersive environments.

\section{KEYWORDS}

Blended Learning; Educational Innovation; Higher Education; Second Life; Student Satisfaction; Kano's Model.

\section{INTRODUCCIÓN}

En el modelo de educación superior que postula la declaración de Bolonia se indica la necesidad de cambios importantes, tanto en las metodologías docentes como en los recursos didácticos empleados por parte del profesorado (Guerra, González y García, 2010). Todo esto es promovido por la existencia de diferentes modalidades de educación, que buscan favorecer el aprendizaje valiéndose de las facilidades inherentes a las TIC, destacando entre sus ventajas la posibilidad de brindar y recibir educación a distancia, la posibilidad de personalizar los contenidos, la baja dependencia de espacios físicos, etc. En este cambio de paradigma, Imbernón, Silva y Guzmán (2011) identifican que las nuevas tecnologías permiten que el alumnado disponga de herramientas y recursos que consiguen que el aprendizaje sea más interactivo y significativo, logrando que se desarrolle en un ambiente más dinámico. Como consecuencia, el número de asignaturas que pueden seguir de forma virtual, o semivirtual, utilizando diferentes plataformas como WebCT, Moodle u otras, aumenta de forma exponencial cada curso, ya que estas herramientas facilitan el desarrollo de metodologías centradas en el E-Learning, dando respuesta a las necesidades y demandas de un gran número de estudiantes (Hinojo, Aznar y Cáceres, 2009).

Sin embargo, el trabajo realizado por Gámiz-Sánchez y Gallego-Arrufat (2016), donde se analiza la opinión de un grupo de estudiantes de educación superior en relación a las metodologías didácticas semipresenciales, pone de manifiesto que la mayor parte del alumnado que participó en la investigación prefería un modelo de aprendizaje híbrido que combine la enseñanza presencial y virtual. Asimismo dicho grupo de discentes identificó como un aspecto negativo la pérdida del trato personal, e implícitamente la presencialidad en un aula. Ante dicha coyuntura, la incorporación de entornos inmersivos 3D podría ayudar a paliar esa percepción. Por otro lado, estaría la predisposición del profesorado a aplicar estos cambios 
metodológicos, por ejemplo, Martín García, García del Dujo y Muñoz Rodríguez (2014) detectaron que uno de los factores más determinantes para la aplicación de un modelo híbrido de enseñanza-aprendizaje (B-Learning) por parte del profesorado universitario reside fundamentalmente en una percepción positiva de los resultados que se podrían obtener. Igualmente se indica que existe una correlación positiva entre la percepción por parte del profesorado de la disponibilidad de infraestructuras adecuadas para aplicar el modelo B-Learning y la predisposición del mismo a la aplicación de dicho modelo de enseñanza-aprendizaje. Es por ello, que uno de los propósitos de este trabajo es contribuir a avanzar en dicha dirección.

Numerosas instituciones de educación superior han incorporado a sus proyectos de innovación docente el uso de entornos inmersivos 3D, conocidos como mundos virtuales o metaversos. En este contexto, Klastrup (2003, p. 101) define mundo virtual como «una representación persistente que ofrece la posibilidad de interacción sincrónica entre las personas usuarias y entre estas y el mundo, dentro de un espacio diseñado en la forma de un universo navegable». Estos mundos virtuales también se denominan MUVEs (MultiUser Virtual Environments) (Clarke, Dede, Ketelhut y Nelson, 2006), refiriéndose a su utilidad como entornos virtuales que permiten a diferentes estudiantes de forma simultánea (a) el acceso a contextos virtuales, (b) la interacción con artefactos digitales (herramientas visuales), (c) la identificación del concepto de corporeidad de cada participante a través de «avatares», (d) la capacidad de comunicarse con el grupo de iguales, el profesorado y personas que tutorizan y, por último, (e) la posibilidad de participar en experiencias que incorporan acciones acerca de problemas similares a los de contextos del mundo real (Dieterle y Clarke, 2007). Han sido muchas las plataformas que han surgido en los últimos años para la experimentación, interacción y simulación digital de entornos inmersivos 3D (Bowers, Ragas y Neely, 2009), aunque la herramienta empleada en el presente proyecto fue Second Life ${ }^{\circledR}(\mathrm{SL})$, plataforma creada por la empresa Linden Lab (http://www.lindenlab.com/). Se trata de una aplicación WWW donde cualquier persona usuaria, una vez registrada y autorizada, puede acceder de forma gratuita a los espacios virtuales que conforman el universo SL. En nuestro caso particular, el espacio virtual o punto de encuentro entre docentes y discentes (denominados avatares residentes en SL) se denomina «Isla UAL-SEIS» (Universidad de Almería y Sociedad Española de Intervención de Sexología), entorno inmersivo 3D que trata de simular la estructura y disposición del Campus de la Universidad de Almería mediante gráficos por computador (más información en Aguilar y otros, 2016).

SL tiene características únicas que hacen recomendable su uso en la práctica docente (Cheong, 2010; Hinrichs, Hill y Patterson, 2011), especialmente en su modalidad a distancia (Burgess, Slate, Rojas-LeBouef 
y LaPrairie, 2010), debido a que el entorno 3D frente al 2D añade una dimensión espacial en la que las personas usuarias están representadas visualmente como avatares y son capaces de moverse en su entorno (Dickey, 2003; Monahan, McArdle y Bertolotto, 2008). Es precisamente en este contexto educativo, donde SL proporciona un entorno de constructivismo, socialización, exploración, descubrimiento y un espacio de aprendizaje creativo (Burgess y otros, 2010). Esto se ha traducido en un crecimiento y aumento del interés por este metarverso dentro de la comunidad académica, tanto en las escuelas secundarias como en las universidades (Semerano, Carloni, Barelli, Scapigliati y Gianolio, 2008; Hinrichs y otros, 2011; Beltrán, Gutiérrez y Garzón 2012; Cheng, 2014). Además SL presenta la capacidad de potenciar la sensación de co-presencia del grupo de estudiantes e inmersión en un entorno en línea (Warburton, 2009). La participación del alumnado ha sido definida por muchos agentes de la comunidad educativa como la pieza central de la eficacia del aprendizaje (Trindade, Fiolhais y Almeida, 2002; Dickey 2005; Zhang, Zhou, Briggs y Nunamaker, 2006). Sin embargo, como indican Pellas y Kazanidis (2015), dicho aspecto en los entornos virtuales debe combinarse con un escenario más amplio a través de la relación inmersión-motivación.

En referencia a la docencia de materias más técnicas, el trabajo realizado por Garzón, Beltrán y Martínez (2009) puso de relieve que el alumnado de ingeniería prefiere metodologías de aprendizaje más activas y enfocadas a la componente práctica de la asignatura. Por ende, los elementos o recursos que incentiven la motivación ayudarán a que el alumnado esté más activo, participativo y crítico (Wehner, Gump y Downey, 2011).

Wang y Burton (2013) revisaron 107 artículos en revistas revisadas por pares con el fin de explorar cómo se investiga y se aplica SL en la educación. Entre estas publicaciones, 50 eran estudios empíricos, 54 discusiones puramente conceptuales y 3 fueron trabajos de revisión. Es importante señalar que entre los estudios empíricos se contabilizaron trabajos que simplemente describían experiencias en forma de comentarios sin preguntas preestablecidas de investigación y sin seguir ninguna metodología sistemática. El número de publicaciones alcanzó un pico en 2009. A partir de entonces empezó a disminuir, probablemente debido a la trayectoria en forma de cúpula que desarrolla un medio nuevo cuando entra en la escena educativa, generando inicialmente un gran interés que posteriormente va decreciendo (Reiser, 2001).

Teniendo en cuenta dicho marco contextual, la pregunta de investigación se centra en conocer la percepción, tomando como referencia el grado de satisfacción, de un grupo de estudiantes de la Universidad acerca de la enseñanza a distancia a través de la plataforma Second Life: ¿Cuál es 
la valoración del alumnado de ingeniera mecánica en relación a la enseñanza sincrona a distancia a través de entornos inmersivos 3D? Para la elección de las características principales a valorar se ha partido del trabajo previo realizado por el grupo docente en esta línea de estudio (Aguilar y otros, 2016). El objetivo final es la evaluación, por medio de una cuantificación factible y efectiva, de la satisfacción del alumnado en relación tanto con la interfaz del entorno inmersivo 3D empleado como con el propio método de enseñanza síncrona a distancia.

\section{MATERIAL Y MÉTODOS}

El desarrollo del estudio se ha llevado a cabo a lo largo de tres fases diferenciadas. En la primera fase, fase preparatoria, se diseñó y planificó el procedimiento seguido en el desarrollo de la investigación experimental. En la segunda fase, fase exploratoria, se llevaron a cabo las diferentes sesiones programadas en el marco del proyecto y se procedió a la obtención de las valoraciones del alumnado. En la tercera y última fase, fase final, se aplicó y adaptó el conocido modelo de Kano (Kano, Seraku, Takahashi y Tsuji, 1984), diseñado para clasificar tipos de atributos de calidad con diferente impacto en la satisfacción de las personas usuarias con el fin de poder desarrollar y mejorar productos y/o servicios. El modelo de Kano ha sufrido revisiones a lo largo del tiempo, siendo aplicado en distintos ámbitos. Por ejemplo, fue aplicado para la evaluación de la calidad del servicio en los hipermercados (Ting y Chen, 2002), en la industria del automóvil (Matzler, Bailom, Hinterhuber, Renzl y Pichler, 2004) o en un servicio de comunidades virtuales en la web (Kuo, 2004).

\section{Fase preparatoria}

En esta primera fase, y como estudio preliminar, se realizó una revisión bibliográfica sobre SL y su aplicación en el ámbito educativo.

A continuación se programaron las sesiones virtuales a desarrollar en el entorno inmersivo SL de la «Isla UAL-SEIS», teniendo en cuenta la programación docente de la asignatura Diseño Asistido por Ordenador (DAO) del Grado en Ingeniería Mecánica de la Universidad de Almería, ya que el temario a impartir en dichas sesiones no se volvería a repetir en las clases presenciales «face-to-face». Al mismo tiempo, se preparó un breve tutorial con los aspectos previos necesarios para participar en la experiencia que posteriormente se remitió al grupo de estudiantes para que unas semanas antes tuviesen su primera toma de contacto con la plataforma y crearan su propio avatar (Figura 1). 


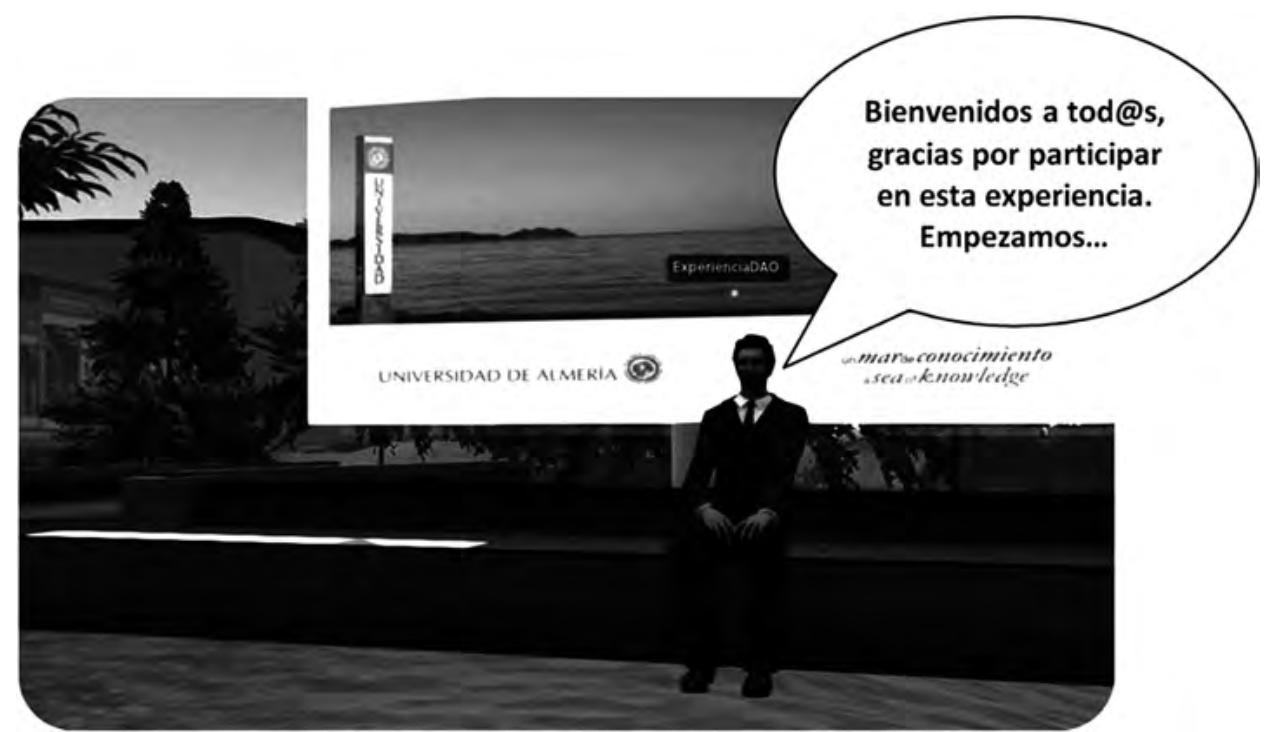

Figura 1. Avatar tutorial «Experiencia DAO» en la Isla UAL-SEIS

Por último, se desarrolló un cuestionario (véase en: https://goo.gl/ fYKXEN) para la evaluación cuantitativa de la satisfacción del alumnado con la Experiencia DAO compuesto por varios ítems y dividido en tres secciones principales (tabla 1): Metodología (M), Interfaz (I) y Adaptabilidad (A). También se incluyó una sección destinada a investigar algunas propuestas que permitiesen mejorar la interfaz y la propia experiencia de enseñanzaaprendizaje (sección de Propuestas (P)). Para la elaboración del cuestionario se tuvieron en cuenta, por una parte, los atributos principales a introducir para conocer las fortalezas y debilidades del recurso docente basado en entornos inmersivos 3D. Por otra, las características propias de evaluación que se requieren para la aplicación posterior del modelo de estimación de la satisfacción de las personas usuarias propuesto por Kano.

\section{Fase exploratoria}

En la fase exploratoria participaron 40 estudiantes de la asignatura Diseño Asistido por Ordenador. El alumnado fue dividido en 3 grupos de trabajo, por lo que la asistencia a cada una de las tres sesiones programadas de docencia vía SL fue de unos 12-15 estudiantes (Figura 2). Cada estudiante asistió a una presentación general del proyecto impartida en la Isla UAL-SEIS, donde se familiarizó con las herramientas de navegación y comunicación. A continuación se celebraron las tres sesiones de docencia SL en relación a contenidos típicos de una clase teórico-práctica de DAO de una hora y media de duración. 
Tabla 1

Ítems a evaluar en la Experiencia DAO

\begin{tabular}{cl}
\hline Ítems & \multicolumn{1}{c}{ Atributos } \\
\hline \multicolumn{1}{c}{ Metodología $(M)$} \\
\hline Ampliación de los recursos formativos (WebCT/Blackboard Learning System) \\
de la asignatura DAO.
\end{tabular}
Interfaz (I)

I1 Fácil navegación y exploración para seguir una clase de DAO.

I2 Buena calidad gráfica y realismo 3D de la Isla UAL-SEIS.

I3 Aspecto de comunicación agradable.

I4 Navegación y exploración en español.

I5 Requisito de un equipo informático y una buena conexión a internet.

I6 Parte de la asignatura se puede seguir desde casa, biblioteca, trabajo... $(24 \mathrm{~h} / 24 \mathrm{~h})$.

\section{Adaptabilidad (A)}

A1 Aumento del interés y la atención en el desarrollo de una asignatura en general.

A2 Aumento del interés por materias que emplean el paradigma «learning by doing» (asignaturas de alto contenido práctico como DAO).

A3 Adaptación a su nivel de conocimientos informáticos.

A4 Necesidad de un buen nivel de percepción y visualización espacial.

A5 Estudiantes con mucha paciencia.

\section{Propuestas $(P)$}

P1 Reuniones sin asistencia del profesorado para trabajar en grupos (por ejemplo sesiones de brainstorming).

P2 Mejora del realismo 3D de la Isla UAL-SEIS. 
Una vez completadas todas las sesiones planificadas, y en una sesión presencial, tras explicar detenidamente el procedimiento para contestar el cuestionario comentado en el apartado anterior, se procedió a la cumplimentación del mismo por parte del grupo de estudiantes participante. La versión final del cuestionario constó de 20 preguntas dobles, una directa y otra complementaria. Tanto las preguntas directas (funcional o positiva) como las complementarias (disfuncional o negativa) tenían cinco opciones de respuesta: «me gusta», «lo esperaba», «me da igual», «puedo tolerarlo», «no me gusta». También se añadieron dos preguntas adicionales en relación a posibles líneas de trabajo futuro. Asimismo, y en la parte final de dicho cuestionario, se añadieron dos cuestiones abiertas para su evaluación cualitativa.

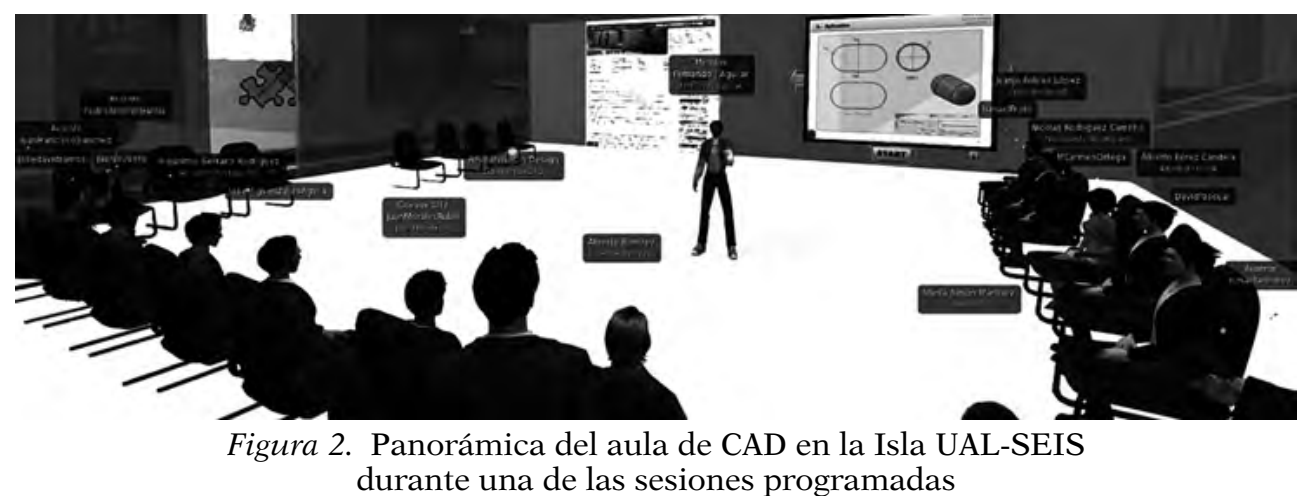

Fase final

En la última fase del proyecto se aplicó una adaptación del modelo conceptual de estimación de la satisfacción de las personas usuarias (en nuestro caso del grupo de estudiantes) en relación a un determinado producto (entorno inmersivo 3D en la enseñanza) desarrollado por Kano. El modelo de evaluación cuantitativa distingue varios tipos de atributos de producto o dimensiones de calidad que influyen en la satisfacción de las personas usuarias de diversas maneras cuando esta los encuentra. A continuación se describe cada uno de los atributos en el contexto del presente estudio:

- Atributos atractivos «A»(attractive): Estos atributos son las características del recurso docente que tienen una mayor influencia en la satisfacción del alumnado. Los atributos atractivos ni son expresados explícitamente ni son esperados por el alumnado. Satisfacer estos atributos conduce a una satisfacción más que proporcional. Si no se encuentran, sin embargo, no hay sensación de insatisfacción. 
- Atributos unidimensionales «O»(one-dimensional): Con respecto a estos atributos, la satisfacción del alumnado es proporcional al nivel del cumplimiento de ellos. Cuanto más alto es el nivel del cumplimiento, más alta es la satisfacción del alumnado y viceversa. Los atributos unidimensionales generalmente son exigidos explícitamente por el alumnado.

- Atributos obligatorios «M» (must-be): Si estos atributos no se satisfacen o no se cumplen, el alumnado se sentirá extremadamente insatisfecho. Por otra parte, como el alumnado toma estos atributos como ya presentes, el cumplimiento de ellos no aumentará su satisfacción. Los atributos obligatorios son los requisitos básicos o mínimos de la herramienta o metodología docente evaluada. La satisfacción de los atributos obligatorios conducirá al alumnado solamente a un estado de no percibir insatisfacción. En efecto, el alumnado percibe los atributos obligatorios como requisitos previos y los asigna como concedidos, por lo que no siente la necesidad de exigirlos explícitamente. Los atributos obligatorios son, en todo caso, un factor competitivo decisivo. Si no se satisfacen, es muy probable que el alumnado no esté interesado en el recurso/ metodología.

- Atributos indiferentes «I» (indifferent): Son aquellos cuya presencia o ausencia no contribuye ni a aumentar ni a disminuir la satisfacción del alumnado con la herramienta docente evaluada.

- Atributos opuestos «R» (reverse): Son características del recurso docente a evaluar que no solo no son deseadas por el alumnado sino que incluso espera lo opuesto.

- Atributos cuestionables «Q» (questionable): Suelen producir valoraciones contradictorias, por lo que generalmente las respuestas no se clasifican en esta categoría. Las cuestiones (funcional y disfuncional) cuya respuesta puede clasificarse como cuestionable están indicando que la pregunta fue expresada incorrectamente, o que el alumnado entendió mal la pregunta o señaló una respuesta incorrecta por error.

La evaluación de la experiencia se ha desarrollado mediante la combinación de las dos respuestas a las preguntas «funcional» (directa) y «disfuncional» (complementaria) según se indica en la tabla 2. Con dicho método se obtiene una clasificación de los atributos en 6 categorías descritas en el apartado anterior (A, O, M, I, R, Q). Para cada atributo, la clasificación está determinada por la categoría que tiene una mayor frecuencia en las respuestas. Si dos o más ítems tienen un mismo resultado, se aplica la siguiente regla $\mathrm{M}>\mathrm{O}>\mathrm{A}>\mathrm{I}$ (en base a la clasificación que tendría el mayor impacto sobre la evaluación de la herramienta de aprendizaje propuesta). 
Tabla 2

Tabla de evaluación de Kano

\begin{tabular}{c|l|ccccc}
\hline \multicolumn{2}{c|}{} & \multicolumn{5}{c}{ Pregunta complementaria (negativa) } \\
\cline { 3 - 7 } \multicolumn{2}{c|}{} & $\begin{array}{c}\text { Me } \\
\text { gusta }\end{array}$ & $\begin{array}{c}\text { Lo } \\
\text { esperaba }\end{array}$ & $\begin{array}{c}\text { Me da } \\
\text { igual }\end{array}$ & $\begin{array}{c}\text { Puedo } \\
\text { tolerarlo }\end{array}$ & $\begin{array}{c}\text { No me } \\
\text { gusta }\end{array}$ \\
\hline \multirow{3}{*}{$\begin{array}{c}\text { Pregunta } \\
\text { directa }\end{array}$} & Me gusta & $\mathrm{Q}$ & $\mathrm{A}$ & $\mathrm{A}$ & $\mathrm{A}$ & $\mathrm{O}$ \\
(positiva) & Lo esperaba & $\mathrm{R}$ & $\mathrm{I}$ & $\mathrm{I}$ & $\mathrm{I}$ & $\mathrm{M}$ \\
& Me da igual & $\mathrm{R}$ & $\mathrm{I}$ & $\mathrm{I}$ & $\mathrm{I}$ & $\mathrm{M}$ \\
& Puedo tolerarlo & $\mathrm{R}$ & $\mathrm{I}$ & $\mathrm{I}$ & $\mathrm{I}$ & $\mathrm{M}$ \\
& No me gusta & $\mathrm{R}$ & $\mathrm{R}$ & $\mathrm{R}$ & $\mathrm{R}$ & $\mathrm{Q}$ \\
\hline
\end{tabular}

Con el fin de ampliar los resultados obtenidos a partir de la categorización de los atributos y profundizar en el análisis de la satisfacción del alumnado con la experiencia docente realizada mediante el empleo de entornos inmersivos 3D, hemos tomado como referencia el trabajo realizado por Violante y Vezzetti (2013). Que aplican el modelo de evaluación de la calidad de Kano para medir la satisfacción de un grupo de estudiantes en relación con la enseñanza de una aplicación médica en modalidad E-Learning. Con el propósito de pormenorizar qué atributos de calidad podían presentar una mayor influencia en la satisfacción del alumnado, calcularon el índice de satisfacción establecido por Berger y otros (1993). El índice CS (coeficiente de satisfacción) es indicativo de la fuerza con la que una característica del producto puede influir en la satisfacción o, en caso de "no cumplimiento», insatisfacción de las personas usuarias.

Para calcular los coeficientes de satisfacción (CS) e insatisfacción (CI) se han aplicado las siguientes expresiones:

$$
\begin{gathered}
C S=\frac{A+O}{(A+O+M+1)} \\
C I=\frac{O+M}{(A+O+M+1)(-1)}
\end{gathered}
$$

Nota. A, O, M e I representan el número de respuestas de cada estudiante clasificadas como atributos atractivos (A), unidimensionales (O), obligatorios (M) o indiferentes (I).

Para la interpretación de los resultados habrá que tener en cuenta que el grado de satisfacción varía de 0 a 1 . Si el valor de CS es cercano a 1 significa que la propuesta tiene un efecto claramente positivo en el aumento de la satisfacción del alumnado. Por otra parte, el grado de insatisfacción 
varía entre 0 y -1 . Si el valor de $\mathrm{CI}$ es cercano a -1 significa que la propuesta produce claramente insatisfacción en el alumnado.

\section{ANÁLISIS Y RESULTADOS}

Los resultados de este trabajo se organizan a continuación según las tres dimensiones principales evaluadas (metodología, interfaz y adaptabilidad). Se añade además una cuarta dimensión relacionada con la percepción del alumnado sobre posibles líneas de mejora del método propuesto. Posteriormente se incorporan y analizan en detalle los valores obtenidos para los coeficientes CS y CI. Por último, y formando parte de una tercera sección, se realiza una valoración a partir del análisis de las dos preguntas abiertas formuladas en el cuestionario para permitir contrastar cualitativamente los resultados cuantitativos ofrecidos por la adaptación del modelo de Kano empleada en este artículo.

\section{Evaluación cuantitativa de la satisfacción del alumnado}

\section{Dimensión Metodología}

Aspectos como la ampliación de los recursos formativos [M1], la complementación de la docencia tradicional de tipo presencial con actividades interactivas en SL [M2], la posibilidad de impartir temas teóricos mediante entornos inmersivos 3D como alternativa a la docencia face-to-face [M3], o el plus de innovación aportado por la plataforma de enseñanza síncrona a distancia basada en SL [M6], parecen ser percibidos por el alumnado como cualidades atractivas (predomina la frecuencia de respuestas clasificadas en la categoría A) en relación al recurso docente evaluado en este trabajo (tabla 3 ).

Por otra parte, el grupo de estudiantes considera como atributos unidimensionales el que el recurso docente facilite la adquisición de los conocimientos técnicos [M7], que refuerce y/o amplíe los conocimientos sobre el Diseño Asistido por Ordenador [M8], y que estimule el desarrollo de habilidades relacionadas con la creatividad [M9] (tabla 3). Como indicábamos anteriormente, los atributos unidimensionales son aquellos que, cuando se presentan en el producto, se traducen rápidamente en satisfacción de la personas usuaria y viceversa. En este sentido suelen ser características del producto claramente exigidas.

Finalmente, el alumnado categoriza la propuesta de que un tema práctico se imparta a través de SL, a modo de alternativa a la clase de 
prácticas [M4], como una cualidad opuesta (R) (tabla 3), es decir, que no solo no lo desean sino que incluso esperan lo opuesto (que la clase práctica sea presencial y se imparta en el Laboratorio de CAD).

\section{Dimensión Interfaz.}

Con respecto a la interfaz del entorno inmersivo 3D empleado en este trabajo (Isla UAL-SEIS en SL), el alumnado considera atractivo (categoría A) que la navegación y la exploración sea en español [I4], así como que parte de la asignatura se pueda seguir desde otro lugar distinto al aula física [I6] (tabla 3). Además les genera satisfacción (categoría O), pero no atracción, una fácil navegación y exploración [I1], y un aspecto de comunicación agradable [I3]. Debido a diferentes inconvenientes técnicos puestos de manifiesto durante el desarrollo de la experiencia, el requisito de un equipo informático apropiado y una buena conexión a internet [I5] queda definido por parte de los participantes como una característica del tipo no deseable u opuesta ( $\mathrm{R}$; «reverse»), y por lo tanto desearían que no fuese un requisito obligatorio para llevar a cabo este tipo de actividad docente.

\section{Dimensión Adaptabilidad}

Es de destacar que el grupo de discentes valore como algo atractivo que la propuesta aumente el interés por materias que emplean el paradigma «learning by doing» [A2] (tabla 3), aunque, como se ha señalado anteriormente, en el caso de la componente de la clase fundamentalmente práctica prefieren la presencialidad y el contacto directo con el profesorado en el contexto del aula de CAD. También valoran satisfactoriamente el aumento de interés y atención [A1] que les genera la nueva herramienta docente indistintamente del tipo de asignatura para la que se emplee. Igualmente valoran satisfactoriamente que el recurso académico se adapte a su nivel de conocimientos informáticos [A3]. El alumnado rechaza la necesidad de tener un buen nivel de percepción y visualización espacial [A4], o que se requiera tener una cierta paciencia para manejarse con destreza en el entorno de SL [A5]. En efecto, del análisis de sus respuestas se deduce justo lo contrario. 
Tabla 3

Evaluación cuantitativa de la satisfacción del alumnado en relación a la Experiencia DAO. Versión adaptada del modelo de Kano

\begin{tabular}{|c|c|c|c|c|c|c|c|}
\hline Ítems & $\mathbf{A}$ & $\mathbf{O}$ & $\mathbf{M}$ & $\mathbf{I}$ & $\mathbf{R}$ & $\mathbf{Q}$ & Categoría dominante \\
\hline \multicolumn{8}{|c|}{ Metodología (M) } \\
\hline M1 & 45 & 10 & 0 & 40 & 5 & 0 & A \\
\hline M2 & 50 & 10 & 3 & 28 & 10 & 0 & A \\
\hline M3 & 45 & 5 & 0 & 35 & 15 & 0 & A \\
\hline M4 & 25 & 8 & 0 & 18 & 50 & 0 & $\mathrm{R}$ \\
\hline M5 & 35 & 13 & 0 & 43 & 10 & 0 & I \\
\hline M6 & 40 & 18 & 3 & 35 & 5 & 0 & A \\
\hline M7 & 25 & 40 & 10 & 20 & 5 & 0 & $\mathrm{O}$ \\
\hline M8 & 35 & 35 & 8 & 18 & 5 & 0 & $\mathrm{O}$ \\
\hline M9 & 30 & 40 & 3 & 25 & 3 & 0 & $\mathrm{O}$ \\
\hline \multicolumn{8}{|c|}{ Interfaz (I) } \\
\hline I1 & 18 & 33 & 20 & 23 & 5 & 3 & $\mathrm{O}$ \\
\hline I2 & 10 & 8 & 8 & 63 & 13 & 0 & I \\
\hline I3 & 23 & 48 & 15 & 13 & 3 & 0 & $\mathrm{O}$ \\
\hline I4 & 48 & 23 & 5 & 18 & 5 & 3 & A \\
\hline I5 & 3 & 15 & 3 & 28 & 53 & 0 & $\mathrm{R}$ \\
\hline I6 & 53 & 35 & 3 & 10 & 0 & 0 & A \\
\hline \multicolumn{8}{|c|}{ Adaptabilidad (A) } \\
\hline $\mathrm{A} 1$ & 28 & 35 & 10 & 25 & 3 & 0 & $\mathrm{O}$ \\
\hline $\mathrm{A} 2$ & 35 & 23 & 5 & 35 & 3 & 0 & A \\
\hline A3 & 35 & 40 & 13 & 13 & 0 & 0 & $\mathrm{O}$ \\
\hline A4 & 10 & 3 & 8 & 33 & 45 & 3 & $\mathrm{R}$ \\
\hline A5 & 0 & 0 & 5 & 43 & 50 & 3 & $\mathrm{R}$ \\
\hline \multicolumn{8}{|c|}{ Propuestas $(P)$} \\
\hline $\mathrm{P} 1$ & 28 & 3 & 0 & 58 & 13 & 0 & $\mathrm{I}$ \\
\hline $\mathrm{P} 2$ & 48 & 28 & 3 & 23 & 0 & 0 & A \\
\hline
\end{tabular}

Nota. Frecuencia de respuestas para cada categoría expresada en \%.

\section{Dimensión Propuesta}

En relación a posibles líneas futuras de mejora del método docente evaluado, el grupo de participantes en la Experiencia DAO consideraría un aspecto atractivo el hecho de que se realizara un sobreesfuerzo para mejorar el realismo 3D de la Isla UAL-SEIS [P2]. Hay que tener en cuenta que esta 
valoración, a diferencia de la evaluación de las tres dimensiones anteriores, se efectúa en base a una hipótesis sin materializar.

\section{Índices de satisfacción e insatisfacción en relación al método docente evaluado}

En este apartado se ha abordado la representación gráfica del grado de satisfacción o insatisfacción del alumnado que ha participado en la experiencia (Figura 3). Para la interpretación de los resultados hay que tener en cuenta que cada atributo o característica evaluada, debido a la propia metodología empleada (Berger y otros, 1993), da lugar a dos coeficientes que miden percepciones de personas usuarias totalmente contrarias pero relacionadas. En este sentido, la lectura final de la valoración de cada atributo depende de un análisis conjunto de la satisfacción e insatisfacción provocada en la persona usuaria más que de una evaluación por separado. Esta estrategia de análisis de resultados mejora la capacidad de elección de un atributo sobre otro en el momento del desarrollo de un producto (en nuestro caso de la herramienta docente propuesta) y permite seleccionar, de una forma sistemática y a partir de los resultados obtenidos en las encuestas, los atributos que logran una óptima satisfacción de la persona usuaria.

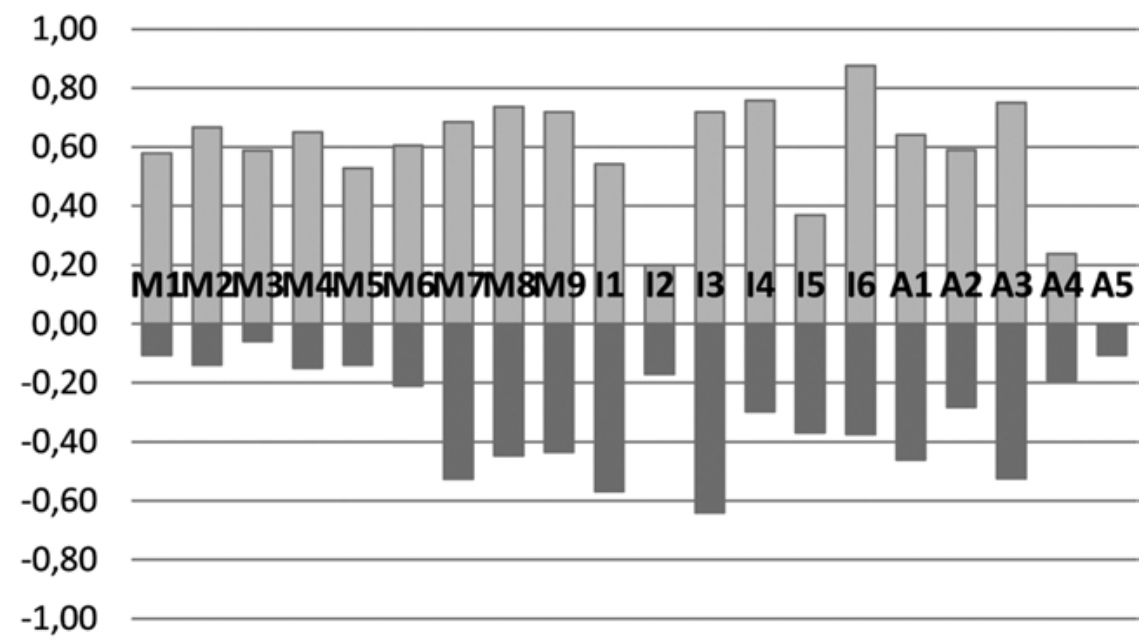

Coeficiente de Satisfacción (CS) — Coeficiente de Insatisfacción (CI)

Figura 3. Representación gráfica del grado de satisfacción del alumnado

A modo de ejemplo, que la interfaz tenga un aspecto de comunicación agradable (pregunta I3) presenta un CS en torno a 0.7 y un CI inferior a -0.6 (Figura 3). Esto significa que esta característica del método docente 
evaluado produce una alta satisfacción en el alumnado. Por el contrario, si no se cumpliera produciría una elevada insatisfacción. Esto permite inferir que este atributo es valorado profundamente por el grupo de discentes, lo que hace imprescindible su inclusión como requisito a tener en cuenta en el desarrollo del recurso docente presentado en este trabajo.

\section{Evaluación cualitativa de la satisfacción del alumnado}

En el cuestionario se añadieron dos preguntas abiertas con la intención de validar/contrastar los resultados cuantitativos obtenidos a partir de la aplicación del modelo de Kano con un análisis de naturaleza cuantitativa. El objetivo fue conocer los pros y contras señalados por el grupo de participantes en relación al uso de SL como herramienta de aprendizaje. Para ello, en este apartado se realiza un análisis de las transcripciones de los comentarios realizados por el alumnado que confirma que el grupo de participantes valora activamente la utilidad y el plus de innovación que incorpora la herramienta docente propuesta en el desarrollo de la asignatura. También ponen de relieve que el método docente basado en entornos inmersivos 3D se presenta como una herramienta complementaria, más que sustitutiva, en relación a la docencia tradicional. A modo de ejemplo, a continuación se exponen algunas de las aportaciones del alumnado participante:

Describa brevemente, ¿qué le ha aparecido la experiencia Second Life Diseño $C A D$ ?

— «Me ha parecido una buena experiencia. Pienso que es un modo útil de impartir clase a distancia y permitir una mayor accesibilidad a cualquier estudiante de poder recibir las explicaciones.»

- «Algo diferente, innovador y entretenido, incluso, en mi opinión ayuda a estar más atento en clase.»

- «Una buena manera de innovar. Me ha gustado mucho la clase teórica, pero en cuanto a la práctica preferiría un «face-to-face». Me ha parecido buena idea el mantener reuniones previas para explicar los trabajos a desarrollar y todos los recursos que contiene S.L. Como parte negativa diré que aún faltan cosas que mejorar.»

Indique algunas propuestas que crea que mejorarían la experiencia

— «Mayor facilidad de conexión desde cualquier computador o red. Mejora de la apariencia de la Isla UAL-SEIS (gráficos).» 
ÓSCAR GONZÁLEZ-YEBRA, MANUEL A. AGUILAR, FERNANDO J. AGUILAR, MANUEL LUCAS

— «Que se haga una experiencia de inicio presencial, todos juntos físicamente en clase, para aprender el manejo de Second Life.»

- «Haría más frecuentemente clases virtuales para familiarizarnos con estos entornos. Es una herramienta beneficiosa tanto para el alumnado como para el profesorado.»

\section{DISCUSIÓN Y CONCLUSIONES}

En una primera etapa preliminar, realizada por el Grupo de Innovación Docente de la Universidad de Almería (Aguilar y otros, 2016), se demostró el potencial de los entornos inmersivos 3D como complemento de plataformas LMS (Learning Management Systems) para el desarrollo de una oferta formativa basada en el aprendizaje B-Learning. En efecto, se trata de sustituir parte de las clases «face-to-face» por una nueva modalidad de enseñanza síncrona donde el punto de encuentro resulta ser un aula virtual más que un aula física. En esta segunda etapa del proyecto de innovación docente se han evaluado cuantitativamente las posibilidades de dicha herramienta, centrándonos en este caso en una propuesta académica para el aprendizaje de materias relacionadas con la Expresión Gráfica en la Ingeniería y el Desarrollo de Productos, área de conocimiento que presenta un balance equilibrado entre las componentes teórica y práctica. En ese sentido, y dando respuesta a la pregunta de investigación planteada inicialmente en este estudio, la valoración del grupo de estudiantes de ingeniería mecánica en relación a la docencia a través de SL de la componente teórica ha sido ampliamente positiva, inclinándose por una docencia presencial tipo «faceto-face» en el caso de la docencia de la componente puramente práctica o aplicada (docencia en aula de CAD).

En base a los resultados obtenidos podemos establecer que la docencia a través de entornos inmersivos $3 \mathrm{D}$ supone un recurso docente útil a la vez que innovador como complemento de un modelo de aprendizaje B-Learning. Unos resultados similares fueron encontrados por Cheng (2014), que coincide en señalar que la mayoría de personas usuarias de SL como herramienta de aprendizaje se mostraron satisfechas con la experiencia, aunque actualmente no se puede remplazar por completo la comunicación cara a cara entre docentes y discentes. En un escenario similar al de este trabajo, Beltrán y otros (2012) detectan un claro aumento de la motivación del alumnado que emplea SL como vehículo de aprendizaje.

Es necesario destacar que la adaptación metodológica desarrollada en el presente trabajo no buscaba la significancia estadística de la clasificación de cada requerimiento de calidad, sino una evaluación y selección de los atributos más importantes que deberían incluirse en un entorno de 
aprendizaje 3D orientado a la enseñanza B-Learning. Aunque hemos trabajado con una muestra de 40 casos, lo suficientemente representativa como para dar respuesta al objetivo planteado en este estudio, sería conveniente ampliar los resultados obtenidos con experiencias similares a la desarrollada en este proyecto en las que:

- Participaran estudiantes de diferentes disciplinas y niveles académicos.

- Se incluyeran las valoraciones del profesorado.

\section{Principales conclusiones}

- Los resultados de este estudio pueden ser utilizados como una importante referencia para el desarrollo y/o modelados de campus universitarios similares en SL, así como para el diseño de nuevas aplicaciones análogas de aprendizaje interactivo, en especial con asignaturas o materias relacionadas con el «Diseño en la Ingeniería».

- Asimismo, los hallazgos de este estudio pueden ayudar a diseñar estrategias de enseñanza para promover el aprendizaje (B-Learning) a través de un uso efectivo de SL.

\section{Recomendaciones y futuras actuaciones}

A corto plazo sería preciso plantear una experiencia similar a la de este estudio con estudiantes de posgrado, con la intención de detectar posibles diferencias en cuanto a la percepción de la herramienta docente entre estudiantes de grado y posgrado. La incorporación de este tipo de herramientas docentes a distancia en el caso de másteres oficiales permitiría el acceso a las universidades españolas de un gran número de alumnado extranjero, potenciando el tan deseado carácter internacional de los estudios de posgrado en España.

A largo plazo, y en caso de una implantación regulada y sistemática de este tipo de herramientas en la oferta formativa en modalidad B-Learning, sería interesante evaluar si la motivación e innovación que valoran tan positivamente el alumnado se mantiene a lo largo del tiempo, con la intención de avanzar hacia una evaluación longitudinal.

Este trabajo se convierte en un punto de inflexión que abre un amplio campo de posibilidades para experimentar no solo con los aspectos técnicos, metodológicos y académicos, sino también con las sensaciones y 
las emociones. Por ejemplo, podemos imaginar la posibilidad de participarimpartir una clase sobre fundamentos de Expresión Gráfica Arquitectónica desde uno de los conjuntos arquitectónicos de La Alhambra (Granada), o una sesión sobre conceptos de Integración Paisajística (diseño de envolventes, colores, texturas...) desde el Parque Natural de Cabo de Gata (Almería).

\section{NOTAS}

* Este estudio ha sido posible gracias a un contrato predoctoral FPI (primer autor) del «Plan Propio de Investigación» de la Universidad de Almería, y al apoyo recibido por parte de la convocatoria bienal 2014-16 de Grupos Docentes de Innovación. Asimismo, en este apartado se hace imprescindible hacer referencia al grupo de estudiantes de la asignatura «Diseño Asistido por Ordenador» (curso 2015/16) que ha participado en la fase exploratoria, por su buena disposición, su ilusión y su tiempo. Sin su colaboración este trabajo no hubiera sido posible. 


\section{REFERENCIAS BIBLIOGRÁFICAS}

Aguilar, F.J., Lucas, M., Aguilar, M.A., Reca, J., Luque, A., Cardona, A. \& Carrión, J.J. (2016). 3D Immersive Evironments in Higher Education Blearning Implementations. Preliminary Results. En X. Fischer, A. Daidie, B. Eynard y M. Paredes (Eds.). Research in Interactive Design (Vol. 4, pp. 624-628). Switzerland: Springer International.

Beltrán Sierra, L.M., Gutiérrez, R.S. \& Garzón-Castro, C.L. (2012). Second Life as a support element for learning electronic related subjects: A real case. Computers \& Education, 58(1), 291302. 10.1016/j.compedu.2011.07.019

Berger, C., Blauth, R., Boger, D., Bolster, C., Burchill, G., DuMouchel, W. \& Walden, D. (1993). Kano's Methods for Understanding Customer-defined Quality. Center for Quality Management Journal, 2(4), 3-36.

Bowers, K.W., Ragas, M.W. \& Neely, J.C. (2009). Assessing the value of virtual worlds for post-secondary instructors: a survey of innovators, early adopters and the early majority in Second Life. International Journal of Humanities and Social Sciences, 3(1), 40-50.

Burgess, M.L., Slate, J.R., RojasLeBouef, A. \& LaPrairie, K. (2010). Teaching and Learning in "Second Life»: Using the Community of Inquiry (CoI) Model to Support Online Instruction with Graduate Students in Instructional Technology. Internet and Higher Education, 13(1), 84-88. 10.1016/j.iheduc.2009.12.003

Cheng, G. (2014). Exploring Students' Learning Styles In Relation to Their Acceptance and Attitudes Towards Using Second Life in Education: A Case Study in Hong Kong. Computers \&
Education, 70, 105-115. 10.1016/j. compedu.2013.08.011

Cheong, D. (2010). The effects of practice teaching sessions in Second Life on the change in pre-service teachers' teaching efficacy. Computers \& Education, 55, 868-880. 10.1016/j. compedu.2010.03.018

Clarke, J., Dede, C., Ketelhut, D.J. \& Nelson, B. (2006). A design-based research strategy to promote scalability for educational innovations. Educational Technology, 46(3), 27-36.

Dickey, M.D. (2003). Teaching in 3D: pedagogical affordances and constraints of 3D virtual worlds for synchronous distance learning. Distance Education, 24(1), 105-121. 10.1080/01587910303047

Dickey, M.D. (2005). Three-dimensional virtual worlds and distance learning: two case studies of active worlds as a medium for distance education. British Journal of Educational Technology, 36(3), 439-451. 10.1111/j.14678535.2005.00477.x

Dieterle, E. \& Clarke, J. (2007). Multiuser virtual environments for teaching and learning. En M. Pagani (Ed.). Encyclopedia of multimedia technology and networking, 2nd ed. Hershey, PA: Idea Group, Inc.

Gámiz-Sánchez, V. y GallegoArrufat, M.J. (2016). Modelo de análisis de metodologías didácticas semipresenciales en Educación Superior. Educación XX1, 19(1), 3961, doi: 10.5944/educXX1.13946

Garzón, C.L., Beltrán, L.M. y Martínez, P. (2009). Estudio de percepción sobre metodologías de enseñanza de temas de electrónica en programas diferentes 
a Ingeniería Electrónica. Revista Educación en Ingeniería, 8, 93-101. Recuperado de http://goo.gl/VMc7Xp

Guerra, S., González, N. y García-Ruiz, R. (2010). Utilización de las TIC por el profesorado universitario como recurso didáctico. Comunicar, 35, 141148. 10.3916/C35-2010-03-07

Hinojo, F.J., Aznar, I. y Cáceres, M.P. (2009). Percepciones del alumnado sobre el blended learning en la universidad. Comunicar, 33, 165-174. 10.3916/c33-2009-03-008

Hinrichs, R., Hill, V. \& Patterson, D. (2011). Book review: Higher education in Virtual Worlds: Teaching and Learning in Second Life. En C. Wankel y J. Kingsley (Eds.). Information Processing and Management, 47, 143146. 10.1016/j.ipm.2010.04.002

Imbernón, F., Silva, P. y Guzmán, C. (2011). Competencias en los procesos de enseñanza-aprendizaje virtual y semipresencial. Comunicar, 36, 107114. 10.3916/C36-2011-03-01

Kano, N., Seraku, N., Takahashi, F. \& Tsuji, S. (1984). Attractive Quality and Must-Be Quality. The Journal of Japanese Society for Quality Control, 14(2), 39-48

Klastrup, L. (2003). A poetics of virtual worlds. Presentado en el Congreso Digital Arts and Culture-DAC 2003, Melbourne, Australia. Recuperado de http://goo.gl/aB3t8e

Kuo, Y.F. (2004). Integrating Kano's Model into Web-community Service Quality. Total Quality Management \& Business Excellence, 15(7), 925-939. 10.1080/14783360410001681854

Martín García, A.V., García del Dujo, A. y Muñoz Rodríguez, J.M. (2014). Factores determinantes de adopcion de Blended Learning en Educacion
Superior. Adaptación del modelo Utaut. Educación XX1, 17(2), 217-240. 10.5944/educxx1.17.2.11489

Matzler, K., Bailom, F., Hinterhuber, H.H., Renzl, B. \& Pichler, J. (2004). The asymmetric relationship between attribute-level performance and overall customer satisfaction: a reconsideration of the importanceperformance analysis. Industrial Marketing Management, 33(4), 271277. 10.1016/S0019-8501(03)00055-5

Monahan, T., McArdle, G. \& Bertolotto, M. (2008). Virtual reality for collaborative e-learning. Computers \& Education, 50, 1339-1353. 10.1016/j. compedu.2006.12.008

Pellas, N. y Kazanidis, I. (2015). On the value of second life for students' engagement in blended and online courses: A comparative study from the higher education in Greece. Education and Information Technologies, 20(3), 445-466. 10.1007/s10639-013-9294-4

Reiser, R.A. (2001). A history of instructional design and technology: Part I: A history of instructional media. Educational Technology Research and Development, 49, 53-64. 10.1007/BF02504506

Semerano, F., Carloni, A., Barelli, A., Scapigliati, A. y Gianolio, M. (2008). Second Life: a non-conventional way to teach. Resuscitation, 77(1), S21. 10.1016/j.resuscitation.2008.03.071

Ting, S.C. \& Chen, C.N. (2002). The asymmetrical and non-linear effects of store quality attributes on customer satisfaction. Total Quality Management, 13(4), 547-569.

Trindade, J., Fiolhais, C. \& Almeida, L. (2002). Science learning in virtual environments: a descriptive study. British Journal of Educational Technology, 33(4), 471-488. 10.1111/1467-8535.00283 
Violante, M.G. \& Vezzetti, E. (2013). Virtual Interactive E-Learning Application: An Evaluation of the Student Satisfaction. Computer Applications in Engineering Education, 23, 72-91. 10.1002/cae.21580

Wang, F. \& Burton, J.K. (2013). Second Life in education: A review of publications from its launch to 2011. British Journal of Educational Technology, 44(3), 357-371. 10.1111/j.1467-8535.2012.01334.x

Warburton, S. (2009). Second Life in higher education: assessing the potential for and the barriers to deploying virtual worlds in learning and teaching. British Journal of Educational Technology, 40(3), 414426. 10.1111/j.1467-8535.2009.00952.x

Wehner, A.K., Gump, A.W. \& Downey, S. (2011). The Effects of Second Life on the Motivation of Undergraduate Students Learning a Foreign Language. Computer Assisted Language Learning, 24(3), 277-289.

Zhang, D., Zhou, L., Briggs, R. \& Nunamaker, F. (2006). Instructional video in e-learning: assessing the impact of interactive video on learning effectiveness. Information and Management, 43(1), 15-27. 


\section{PERFIL ACADÉMICO Y PROFESIONAL DE LOS AUTORES}

Óscar González-Yebra. Ing. en Diseño Industrial por la Escuela Politécnica Superior de Málaga y MSc. en el programa interuniversitario «Representación y Diseño en Ingeniería y Arquitectura». En la actualidad está adscrito como personal docente e investigador en formación al Área de Expresión Gráfica de la Universidad de Almería. Sus intereses de investigación incluyen la enseñanza-aprendizaje del diseño, la innovación docente, el desarrollo del diseño en el sector agroalimentario, el co-diseño y la ingeniería gráfica.

Manuel A. Aguilar. Dr. Ingeniero Agrónomo por la Universidad de Córdoba desde 2001. En la actualidad es Profesor Titular de Universidad del Departamento de Ingeniería de la Universidad de Almería. Coautor de numerosas publicaciones internacionales relacionadas con las disciplinas de teledetección, fusión de datos espaciales y captura, procesamiento, análisis y extracción de información a partir de datos georreferenciados 2D/3D. Conjuntamente trabaja en el desarrollo, aplicación y evaluación de entornos inmersivos 3D en la educación superior.

Fernando J. Aguilar. Dr. Ingeniero Agrónomo por la Universidad de Córdoba desde 1997. Actualmente es Catedrático del Departamento de Ingeniería de la Universidad de Almería. Es coautor y revisor habitual de numerosas publicaciones internacionales relacionadas con las disciplinas de teledetección, fusión de datos espaciales y captura, procesamiento, análisis y extracción de información a partir de datos georreferenciados 2D/3D. Igualmente trabaja en el desarrollo, aplicación y evaluación de entornos inmersivos 3D en la educación superior.

Manuel Lucas. Dr. Médico de Medicina Familiar y Comunitaria. Sexólogo. Profesor del Máster Oficial en Ciencias de la Sexología de la Universidad de Almería. Profesor del Máster en Sexología de la Universidad de Sevilla, Universidad Camilo José Cela de Madrid, y Universidad de Cuenca (Ecuador). Autor de varios libros de Sexología. Experto en construcción de entornos inmersivos 3D. Trabaja en el desarrollo, aplicación y evaluación de entornos inmersivos 3D en la educación superior.

Dirección de los autores: Universidad de Almería Carretera de Sacramento, s/n La Cañada de San Urbano 04120 Almería E-mail: oglezyebra@ual.es maguilar@ual.es faguilar@ual.es lucasmatheu@ono.com 
Fecha Recepción del Artículo: 18. Marzo. 2016

Fecha Modificación Artículo: 05. Diciembre. 2016

Fecha Aceptación del Artículo: 12. Diciembre. 2016

Fecha Revisión para Publicación: 22. Febrero. 2018 